MODELING, IDENTIFICATION AND CONTROL, 2001, VOL. 22, NO. 2, 65-72

doi:10.4173/mic.2001.2.1

\title{
A Logarithmic-Amplitude Polar Diagram
}

\author{
TROND ANDRESEN
}

Keywords: Logarithmic polar diagram, Nyquist criterion, stability, logarithmic spiral

A polar diagram where the amplitude of the transfer function is on a logarithmic scale, is presented. This gives a one-size-fits-all diagram with no need for zooming in and out, and no need for additional reasoning about infinite-radius encirclements when there are poles on the imaginary axis - as opposed to what is usually neccessary with the standard polar (Nyquist-) diagram. All properties needed for stability considerations are upheld, such as encirclements, gain and phase margins. The path for $s$ in the loop transfer function is carefully chosen with regard to possible poles on the imaginary axis. Small excursions into the right half plane in the form of arcs of different-sized logarithmic spirals result in corresponding large but finite arcs that do not overlap in the logarithmic polar plots.

\section{Introduction}

The standard polar or Nyquist plot is a powerful tool to examine closed-loop stability. But this plot is often difficult to employ when the system is stiff, has poles on the imaginary axis or is open-loop unstable. The large span in amplitudes for the open-loop transfer function over the relevant frequency range forces the user to zoom in and out to inspect different-scale plots of the same system, thus losing the system overview, and making it difficult to correctly count encirclements.

A polar diagram where the amplitude of the open-loop transfer function is plotted on a logarithmic scale, is a very effective means to remedy these problems. We will introduce such a diagram through an example: Consider the transfer function,

$$
h_{0}(s)=\frac{K\left(1+T_{a} s\right)\left(1+T_{b} s\right)}{s\left(1+T_{1} s\right)\left(1+T_{2} s\right)\left(1+T_{3} s\right)\left(1+T_{4} s\right)},
$$

with $T_{1}>T_{2}>T_{a}>T_{3}>T_{4}$. (This transfer function corresponds to entry 14 in Table 9.6 in Dorf and Bishop (1998).) The system is open-loop stable, and will therefore be closed-loop stable following the Nyquist criterion if it displays no net encirclements around the point $(-1,0)$.

We choose numerical values such that

$$
h_{0}(s)=\frac{200(1+3 s)(1+2 s)}{s(1+50 s)(1+10 s)(1+0.5 s)(1+0.1 s)}
$$

This is a fairly stiff system. The MATLAB function nyquist (h0) results in the plot shown in Figure 1. The same graph is shown at successively zoomed scales. The polar curve has a fractal-like self-similarity- "bulges" and corresponding crossings of the negative imaginary axis emerge as we zoom in. The total picture is confusing;

Trond Andresen, Department of Engineering Cybernetics, The Norwegian University of Science and Technology, N-7034 Trondheim, Norway. E-mail: Trond.Andresen@itk.ntnu.no. Tel: +4773 594358. Fax: +4773594399. 

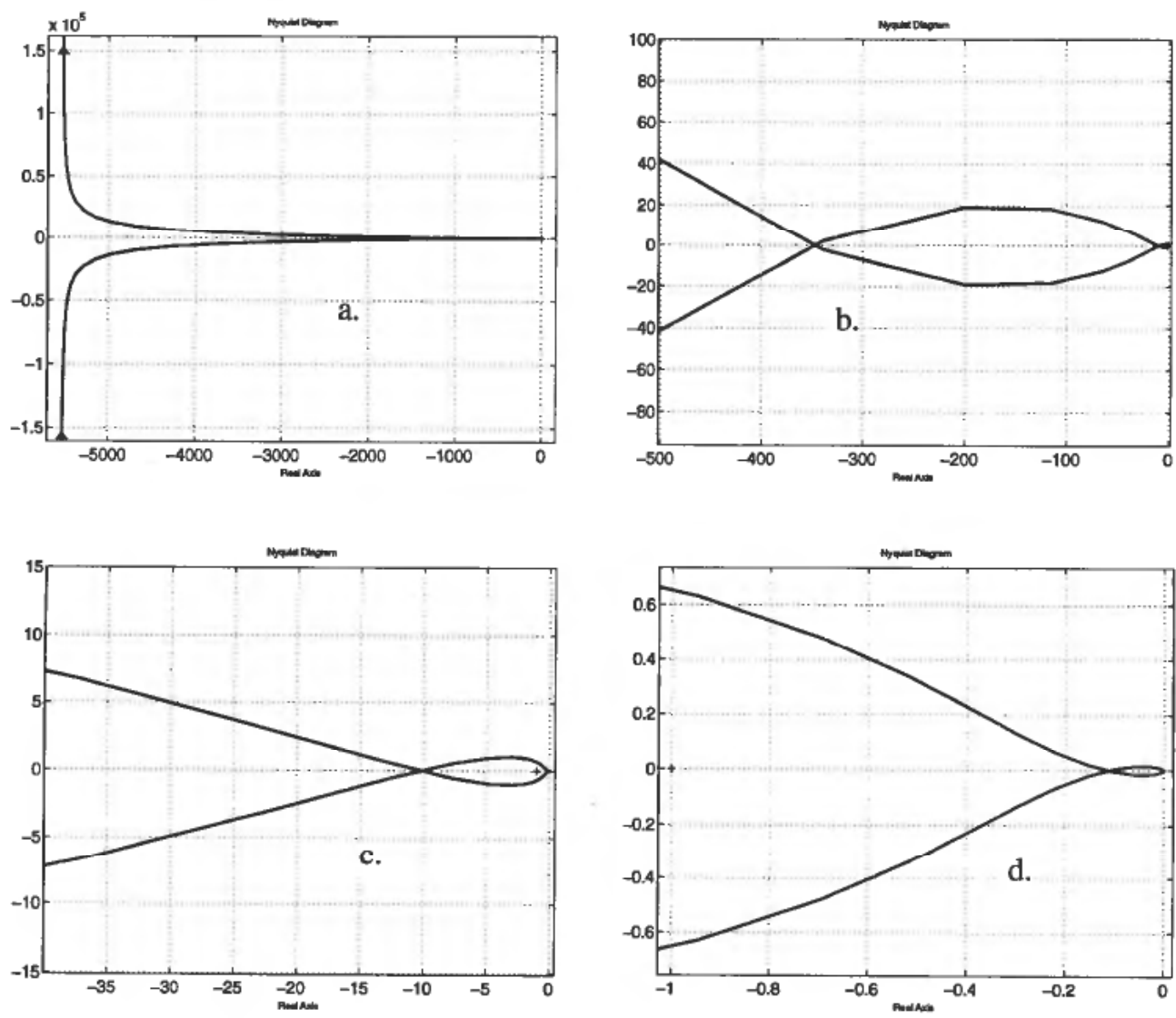

Figure 1. Different enlargements of the polar plot of $h_{0}$.

is the system stable? By zooming in and out and perhaps taking notes, we are able to ascertain that it is indeed stable at the current gain, but that stability is conditional: Starting with a sufficiently small $K$ and increasing, the closed-loop system passes through the stages stable, unstable, stable, unstable.

Now consider Figure 2a. Here the radius vector is plotted not as $\left|h_{0}(s)\right|$, but as $20 \log _{10}\left|h_{0}(s)\right|$, i.e. on a dB scale. Any value lower than $10^{-6}$ is set to $10^{-6}=-120 \mathrm{~dB}$, which is located to the centre of the diagram. The phase angle, $\angle h_{0}(s)$, is the same as in the ordinary polar diagram.

The path for $s$ close to the pole in the origin is chosen as a small half-circular arc into the right half plane, resulting in the large arc for $\left|h_{0}(s)\right|$ in the figure.

We now note that all information needed to immediately establish the stability properties of the closed-loop system can be extracted from Figure 2a. No zooming or alternating between plots are neccessary. And gain and phase margins may be extracted from the figure, except that the gain margin will be in $\mathrm{dB}$. We will from now on call this a $\log$-polar $(L P)$ diagram.

Figure $2 \mathrm{~b}$ shows the LP diagram for $40 \mathrm{~dB}$ higher gain. The corresponding feedback system will now be unstable. 

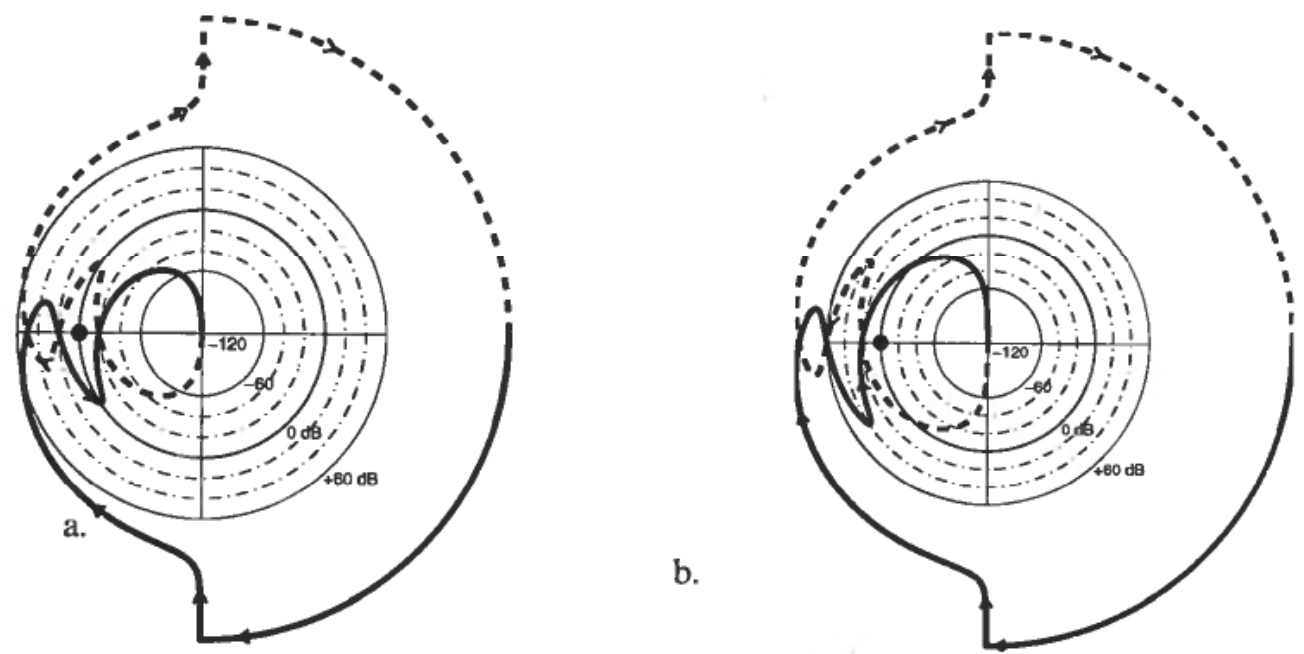

Figure 2. Log-polar plots of $h_{0}$ for gains $K=200$ and 20000.

\section{Implementation of a log-polar MATLAB function}

The LP plot is implemented as an experimental MATLAB function ${ }^{1}$ lognyq (h0 ). We will discuss some of the ideas and problems of this implementation. The case with no poles on the imaginary axis is straightforward. The path for $s$ in $h_{0}(s)$ is chosen with logarithmic spacing from $-j \omega_{\max }$ to $j \omega_{\max }$, where $\omega_{\max }$ is some multiple of the absolute value of the largest pole or zero in $h_{0}(s)$, whichever is the largest.

The case with poles in the origin or somewhere else on the imaginary axis is more complicated. The idea is to make the imagined "infinite (half-) circles" described by $h_{0}(s)$ when $s$ passes imaginary poles, finite. This is achieved by literal use of the textbook argument in connection with presenting the Nyquist criterion for this class of systems: the path of $s$ is given a small detour into the RHP around any pole(s) on the imaginary axis. One must then ensure that the radius $R$ is significantly smaller than the distance from the nearest pole or zero to the imaginary pole in question. This is to avoid the frequency response of $h_{0}(s)$ being noticeably distorted.

At the same time $R$ must not be $t o o$ small, because this will make the corresponding arcs described by $h_{0}(s)$ so large that the interesting part of the LP diagram is dwarfed (see Figure $2 \mathrm{~b}$, where this is slightly the case). This problem is exacerbated if some poles are multiple, since $h_{0}(s)$ is proportional to $R^{-k}$ when $s$ is close to a pole with multiplicity $k$. So in the case $k>1$, the procedure is to first choose $\mathrm{R}$ as if $k=1$, and then substitute $\mathrm{R}$ with $R^{\prime}=R^{1 / k}$, but at the same time ensure that the first condition of $\mathrm{R}$ not being too large, is still satisfied.

A further issue is that arcs of $h_{0}(s)$ may overlap, so that they cannot be distinguished from each other when checking encirclements. This is solved by not using half-circles into the RHP, but half-circular arcs of logarithmic spirals for each pole on the imaginary axis. One such arc of $s$ around a pole in $(0,1)$ is illustrated in Figure 3.

If there are several distinct poles on the imaginary axis, each arc associated with

\footnotetext{
${ }^{1}$ This function may be downloaded as a zipped file containing a MATLAB function. Access http://www.itk.ntnu.no/mic/
} 


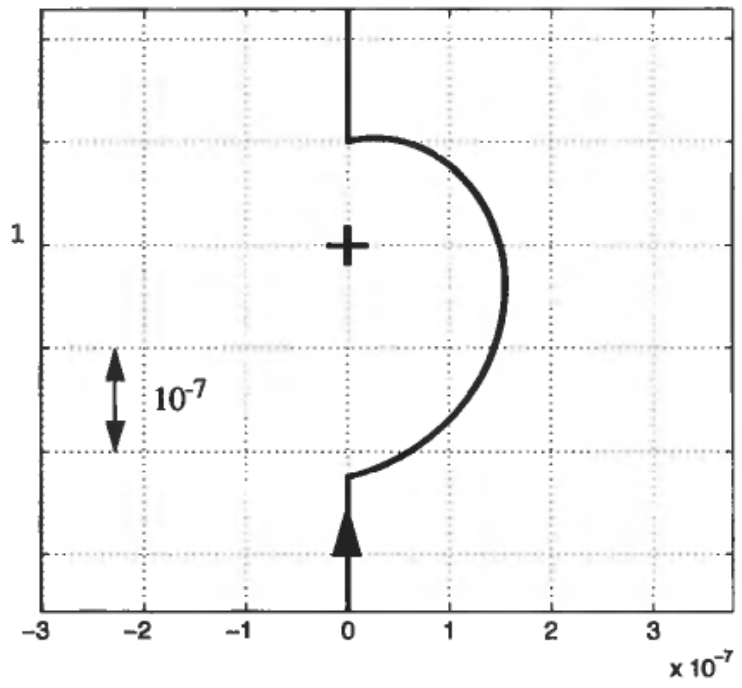

Figure 3. An arc of $s$ around an imaginary pole.
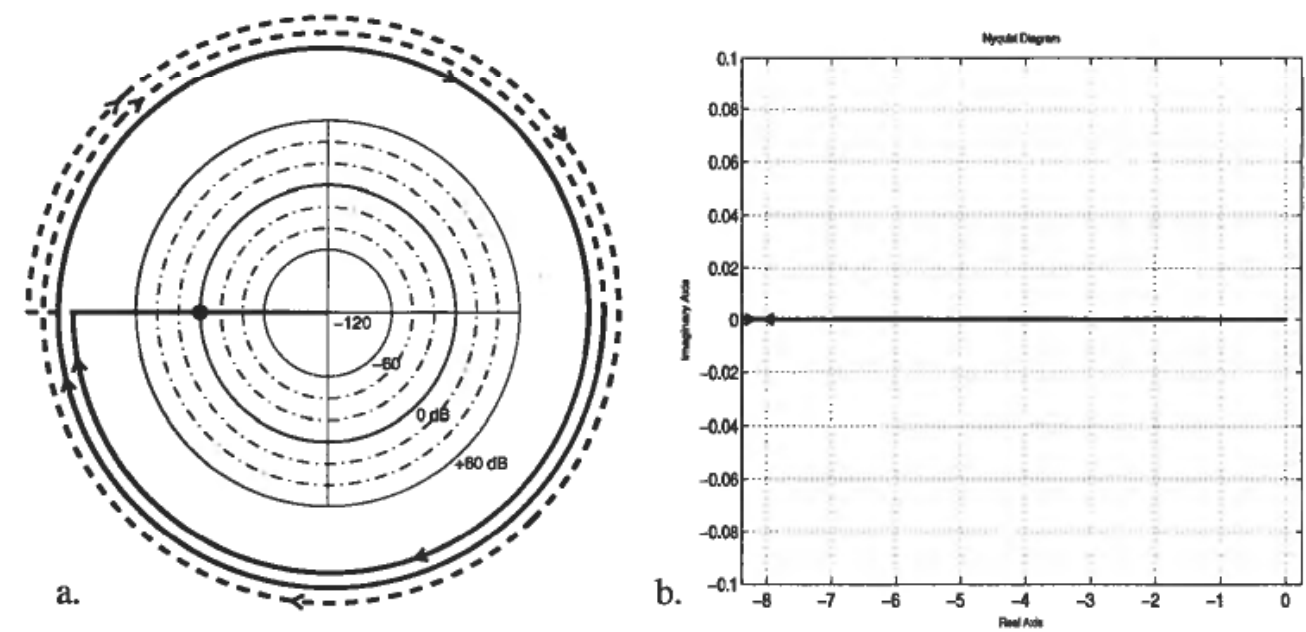

Figure 4. a.: LP plot of $h_{0}(s)=1 / s^{6}$. b.: The corresponding Nyquist plot.

a pole is given a somewhat different size. This ensures that corresponding large arcs in the logarithmic polar plots of $h_{0}(s)$ never overlap, and encirclement counting is easy.

The effect of using logarithmic spirals for the small excursions into the RHP may be illustrated through an LP plot of

$$
h_{0}(s)=\frac{1}{s^{6}}
$$

See Figure 4a. Note that the encircling arcs of have constant distance between them. This is a consequence of the logarithmic scaling of the LP diagram. For small $s$ the logarithmic spiral arc around the origin results in a corresponding inverse which is 

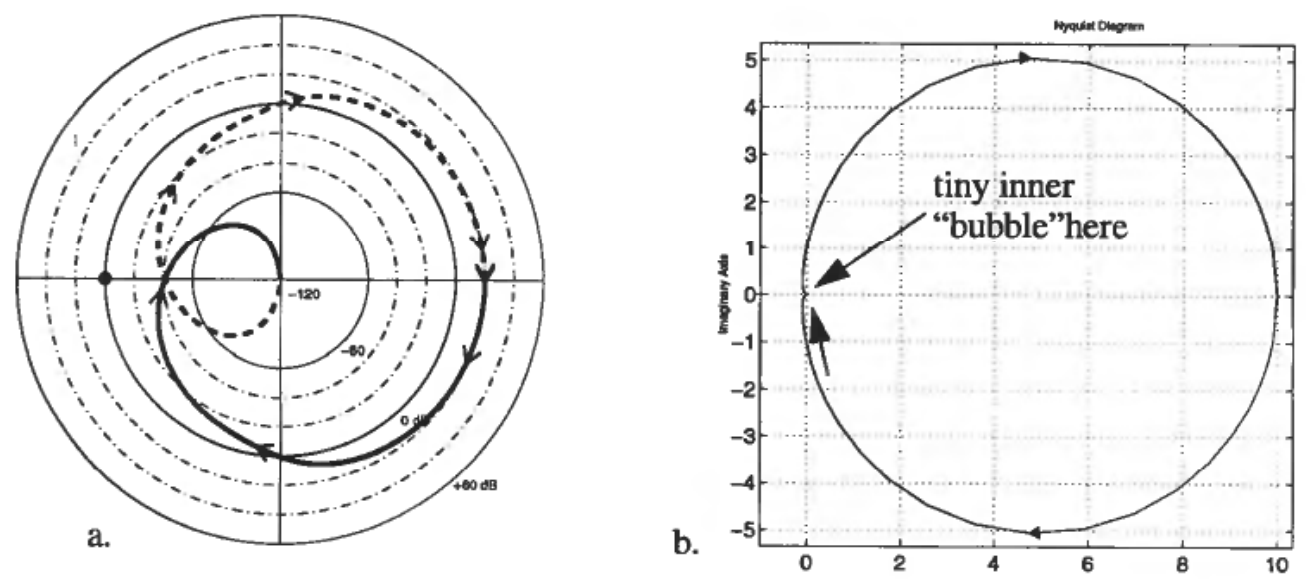

Figure 5. $h_{0}(s)$ corresponding to entry 9.6-3 in Dorf and Bishop (1998).

also a logarithmic spiral, doing three complete turns because the system is of order 6 . The logarithm of the radius vector of a logarithmic spiral gives an Archimedes' spiral (Bronstein and Semendjajew (1966), p. 92) which is characterised by the radius being proportional to the angle; we have $r=k \varphi$. This implies constant distance between LP arcs of $h_{0}(s)$ that comprise several revolutions.

\section{More examples and discussion}

We will illustrate the use of the LP diagram with three additional examples. The first two stem from Dorf and Bishop (1998), with numerical parameter values chosen here. The right-hand part of the figures contains corresponding MATLAB Nyquist plots, not zoomed. The first example is

$$
h_{0}(s)=\frac{10}{(1+1000 s)(1+10 s)(1+s)}
$$

This stiff but simple system is open-loop stable. It becomes closed-loop unstable for large gain. From the LP plot in Figure 5a we see that the gain and phase margins are approximately $40 \mathrm{~dB}$ and $90^{\circ}$ respectively. The inner "bubble" used for these considerations in the LP plot, is so small in the unzoomed Nyquist plot-indicated by arrows- that it disappears.

The next example is shown in Figure 6:

$$
h_{0}(s)=\frac{(1+5 s)(1+s)}{s^{3}}
$$

By inspection of Figure 6a we establish that $h_{0}(s)$ has no net encirclements for the gain shown. If the gain is reduced with around $30 \mathrm{~dB}$ we get two encirclements; the feedback system becomes unstable. One has to zoom in and out in several stages to extract the same information from the corresponding polar plot in Figure $6 \mathrm{~b}$.

The last example is an open-loop slightly unstable system with a time delay. In the time delay case a graphic method is indispensable, since charting of eigenvalues in the feedback system cannot be done.

$$
h_{0}(s)=\frac{(s+0.04)}{s(s+2)(s-0.1)} \mathrm{e}^{-0.5 s}
$$



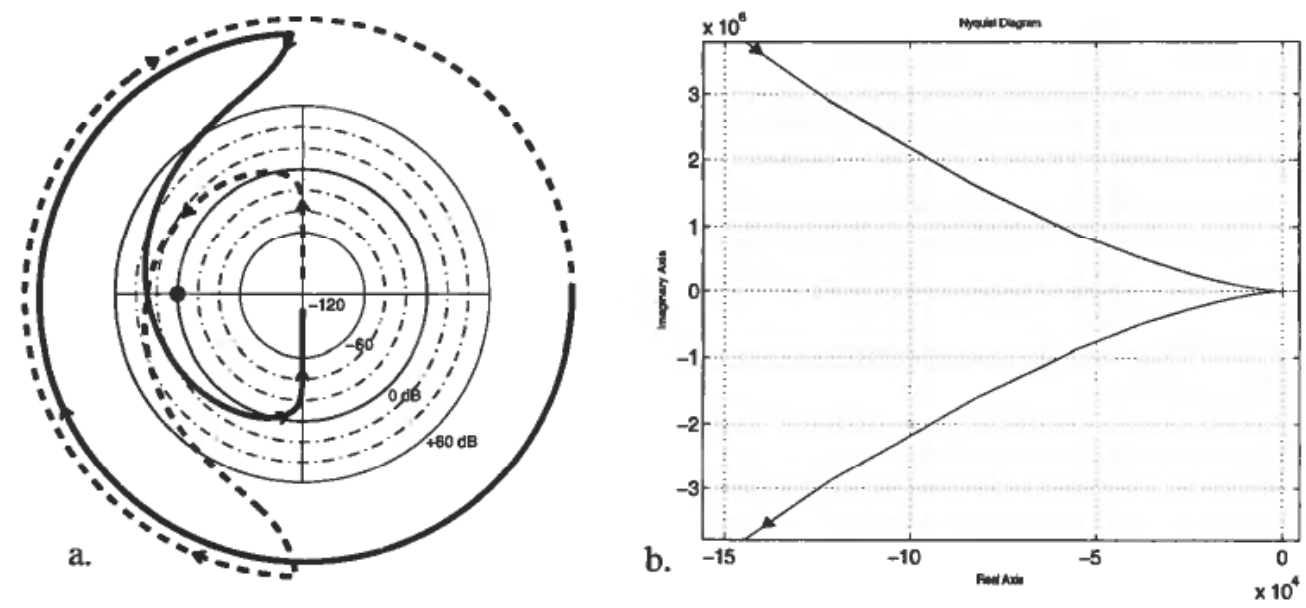

Figure 6. $h_{0}(s)$ corresponding to entry 9.6-13 in Dorf and Bishop (1998).
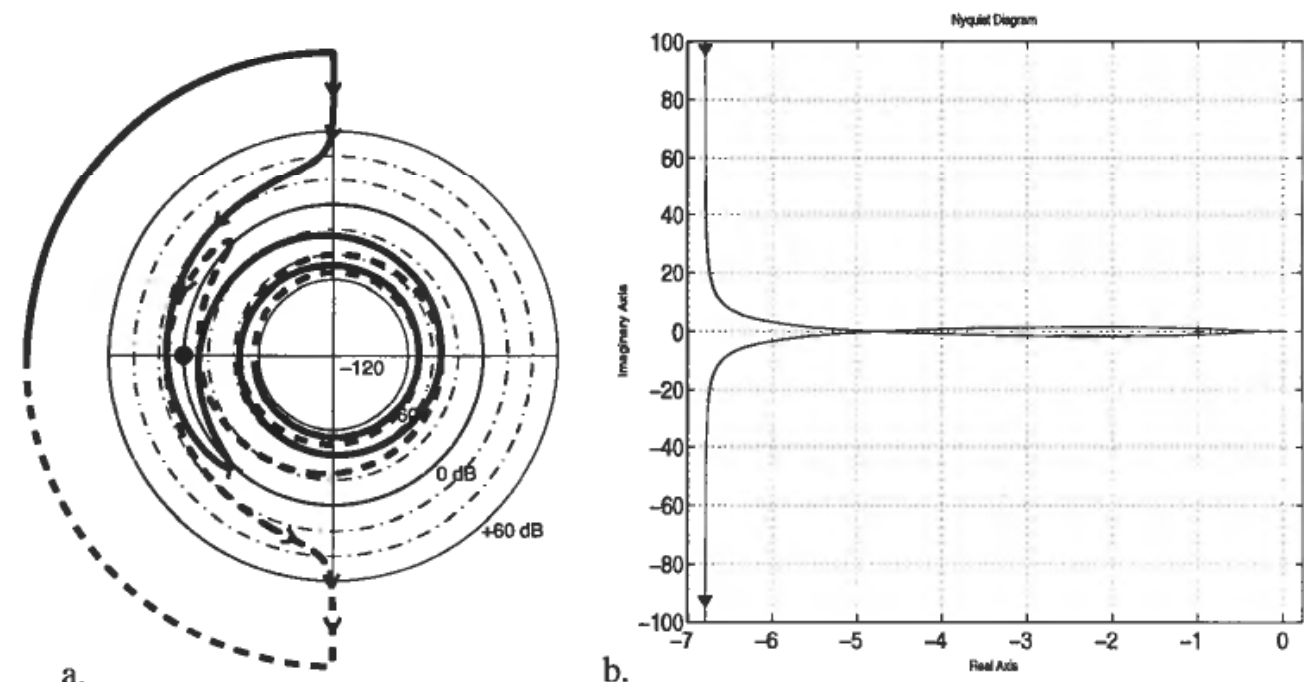

Figure 7. $h_{0}(s)$ as in (6).

Since $h_{0}(s)$ is now open-loop unstable with one pole in the RHP, the Nyquist criterion tells us that the LP plot must encircle $(-1,0)$ counterclockwise one time for closedloop stability. By inspection of Figure 7a we establish that $h_{0}(s)$ satisfies this condition for the gain chosen. Some of the needed information may be extracted from Figure $7 \mathrm{~b}$ without zooming, with some difficulty. But with this polar plot one has to additionally reason out (based on the open-loop pole 0.1 in the RHP) whether the infinite arc due to the pole in the origin goes into the LHP or the RHP. This is not neccessary with the LP plot, since such arcs are finite and therefore clearly shown.

There is some similarity between the plots in Figures 7a and 2a. In both cases we have stability when the $(-1,0)$ point is in the "middle bulge" of the LP plot. But in the 2a case we have no net encirclement, as opposed to for 7a.

Counting net encirclements for higher order systems is facilitated by employing 
the following rule (Bélanger (1995), p. 206): Define clockwise rotation as positive direction. Call the number of encirclements of $h_{0}(s)$ around $(-1,0)$ for $N$. To find $N$, assign an integer to each real-axis crossing to the left of $(-1,0)$. Count clockwise crossings as +1 , anticlockwise crossings as -1 . Sum the integers for all these crossings. The result is $N$. The number of open-loop poles in the RHP is $P$. Then $Z=N+P$, where $Z$ is the number of closed-loop RHP poles.

Since arcs for due to imaginary poles are finite in the LP plot, this counting-ofcrossings rule is easily applied. For the two cases we get:

Figure 2a: $Z=N+P=(-1-1+1+1)+0=0$

Figure 7a: $Z=N+P=(-1-1+1)+1=0$

Finally, a remark on the relation of the LP diagram to another diagram, the log-magnitude-phase curve (Dorf and Bishop (1998), p. 443); in MATLAB plotted with the command nichols (h0). Figure 8a shows the the log-magnitude-phase curve of equation (4).

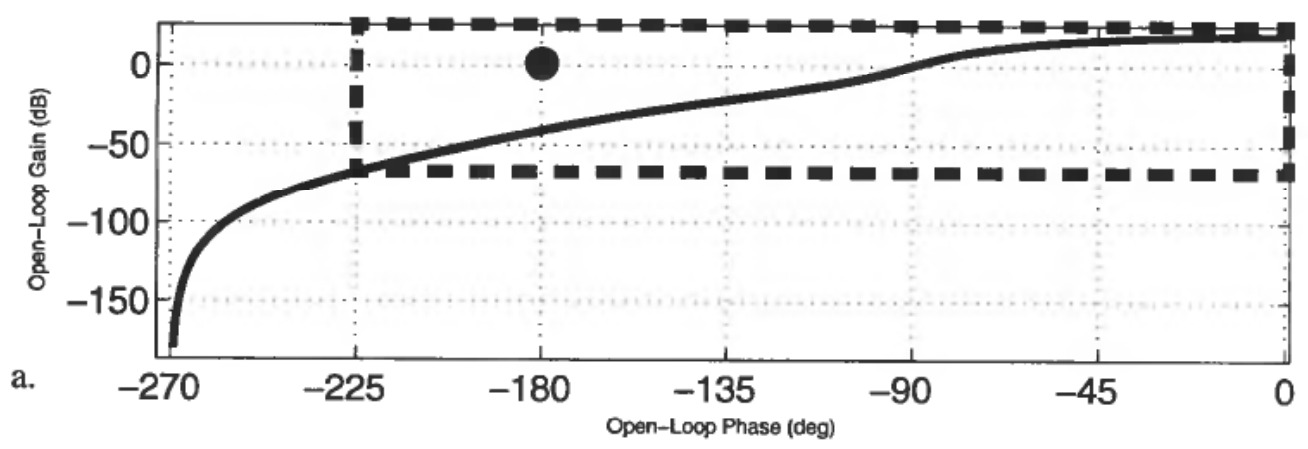

b.

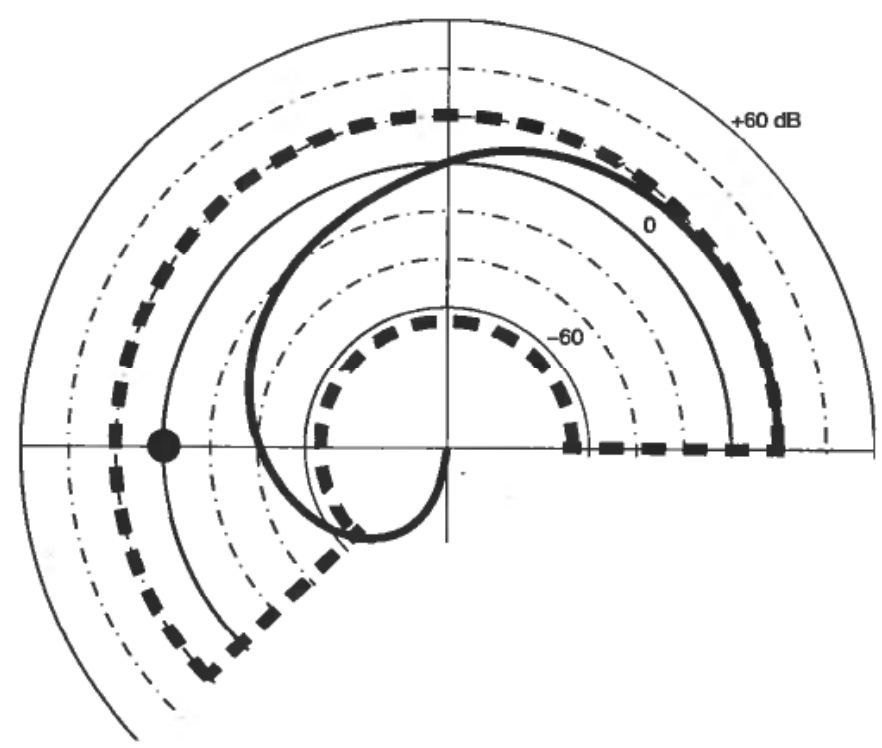

Figure 8. $\quad h_{0}(s)$ as in (4); log-magnitude-phase curve (a.) vs LP plot (b.). 
The dotted rectangle in Figure 8a corresponds to the dotted annulus piece in the LP plot in $8 \mathrm{~b}$. In Figure $8 \mathrm{~b}$ we have used a mirror image of the curve with respect to the real axis, so that decreasing phase from $0^{\circ}$ to $-225^{\circ}$ is now defined in a counterclockwise direction. This is done to facilitate the comparison with Figure 8a.

\section{References}

Dorf, R. C. and Bishop, R. H. (1998). "Modern Control systems", eigth edition, Menlo Park, California: Addison Wesley.

Bronstein, I. N. and Semendjajew, K. A. (1966). "Taschenbuch der Mathematik", Zurich and Frankfurt/Main: Verlag Harri Deutsch.

Bélanger, P. R. (1995). "Control Engineering. A Modern Approach", Florida: Saunders College Publishing, Harcourt Brace and Company. 